$$
\begin{aligned}
& \text { WSP. IP S4-A } \\
& \text { Su su14 }
\end{aligned}
$$

\title{
Fluctuations of Cross Sections Seen in Cosmic RAY DATA?
}

\author{
G.Wilk ${ }^{1 *}$ and Z. Włodarczyk ${ }^{2 \dagger}$ \\ ${ }^{1}$ Soltan Institute for Nuclear Studies, Warsaw, Poland, \\ ${ }^{2}$ Institute of Physics, Pedagogical University, Kielce, Poland
}

\begin{abstract}
We argue that unexpected non-exponential behaviour of some cosmic ray data are just manifestation of cross section fluctuations discussed recently in the literature and observed in nuclear collisions and in the diffraction dissociation experiments on accelerators.
\end{abstract}

PACS: 96.40De, 11.80. La, 12.40. .ig

WSP Kielce, Inst. of Physics preprint - 1/ February 1994

PQ0022139

*e-mail: wilk $\hat{\text { ffuw.edu.pl }}$

${ }^{\dagger}$ e-mail: wsp-fiz'óssrv1.tu.kjelce.pl 
Cosmic ray experiments reported for some time peculiar results concerning the propagation of the initial flux of incoming nucleons (cf. [1,2] for the review of most recent results and earlier references). They can be presented in different ways but for the purpose of the present note we shall concentrate on the example of the distribution of cascade starting points in the extrathick lead chamber of PAMIR experiment [3]. The corresponding data points for the number of cascades originating at depth $T$ (measured in cascade units, 1 c.u. $\left.=6.4 \mathrm{~g} / \mathrm{cm}^{2}=0.56 \mathrm{~cm}\right), d N / d T\left(E_{h}^{\gamma}>6.3 \mathrm{TeV}\right) \mathrm{M}^{-2}$ year ${ }^{-1}$, are shown in Fig. 1 . What is regarded as peculiar or even unexpected is the fact that whereas at small depths (up to $\sim 60 \mathrm{~cm}$ of lead) we observe the usual absorption of hadrons (with absorption length $\lambda_{a b s}=(191 \pm 8) \mathrm{g} / \mathrm{cm}^{2}$ which corresponds to $\sigma_{\text {prod }}^{p+P b}=(2120 \pm 260) \mathrm{mb}$ and agrees within the error with accelerator data at $\left.200 \mathrm{GeV}\right)$, at biggest thickness there is noticeably excess of experimental points above the simple extrapolation of small depths data. It contradicts a natural expectation that any approximation leading to the commonly used formula

$$
\frac{d N}{d T}=\text { const } \cdot \exp \left(-T / \lambda_{a b s}\right)
$$

should be valid also for biggest thickness of lead and observed discrepancy means that original hadrons tend to fly longer without interaction (therefore sometimes a term long flying component is coined for this type of phenomenon [2]).

So far two hypothesis have been widely discussed in the literature $[1,2]$ aiming to explain this observation:

(i) charm particles with $\sigma_{c h a r m-P b}<<\sigma_{F r o d}^{p-P b}$ are produced somewhere in upper parts of chamber;

(ii) there exist some new weakly absorbed hadrons not yet found in accelerators.

The weakness of first hypothesis is that in order to explain the observed effect one needs uncomfortable large value of the cross section for charm production: $\sigma_{\text {charm }} \simeq 3 \mathrm{mb} / \mathrm{nucl}$ at hadron (LAB) energy $E \simeq 150 \mathrm{TeV}$, which is in contradiction with the extrapolation of accelerator data to this energy region. The second hypothesis demands that new "exotic" hadrons should have very large absorption paths $\lambda_{a b s} \simeq 2000 \mathrm{~g} / \mathrm{cm}^{2}$. So far there was no indication of their presence in other experiments.

In this note we would like to propose yet another simple explanation of the abovementioned result, which follows in a sense the idea of second hypothesis but avoids its "exoticity". Namely, we argue that the observed effect can be just manifestation of the fluctuations of the hadronic cross sections discussed recently in the literature and observed in diffraction dissociation experiments on accelerators $[4,5]$. Their origin is traced down to the fact that hadrons have internal degrees of freedom (colour carrying quarks and gluons) and can thus collide in different internal configurations resulting in different cross sections. Refering an interested reader to [5] for details and physical justification of this phenomenon we would like to say here only that it is argued there that hadronic cross sections should be regarded as fluctuating quantities with widths (defined as normalized dispersion)

$$
\omega=\frac{\left\langle\sigma^{2}\right\rangle-\langle\sigma\rangle^{2}}{\langle\sigma\rangle^{2}}
$$

growing logaritmically with energy (at least to $p_{l a b}=300(\mathrm{GeV})$.

To this end we have calculated distributions of depths $T$ (in $\mathrm{Pb}$ ) of first interactions for different $\omega$ 's and compared them in Fig. 1 with Pamir experimental data [1]. It has been done in the following way.

- From the energy distribution of the initial hadronic flux (cf. [6]),

$$
\frac{d N}{d E}=A \cdot E^{-\gamma} ; \quad \gamma=3,
$$

we have sampled the initial energy $E \in\left(E_{t h}=6.3 \mathrm{TeV}, \infty\right)[7]$ and established the corresponding mean cross section $\bar{\sigma}(E)$ and sampled the actual $\sigma$ from the uniform distribution $\sigma(E) \in[(1-$ $\left.\frac{\sqrt{3 \omega}}{}\right) \bar{\sigma},(1+\sqrt{3 \omega}) \bar{\sigma}$ ] (which follows closely the modified Gaussian result of [5], ef. their Fig. 4).

- With such $\sigma$ we have calculated the mean free path in lead, $\lambda=A m_{p} / \sigma$ ( $A$ is the atomic number and $m_{p}$ the nucleon mass) and found the actual point of interaction $x$ from the standard formula for the probability of penetration $P(x)=\exp (-x / \lambda)$. 
- At the same time we have estimated the electromagnetic energy emitted at this point (the only component of the cascade measured in this experiment), $E_{\gamma}=K_{\gamma} \cdot E$, where $K_{\gamma} \in(0,1)$ is the inelasticity sampled from the distribution

$$
\chi\left(K_{\gamma}\right)=\frac{1}{\beta \Gamma(\alpha)} \cdot\left(\frac{K_{\gamma}}{\beta}\right)^{\alpha-1} \cdot \exp \left(-\frac{K_{\gamma}}{\beta}\right),
$$

with $\left\langle K_{\gamma}\right\rangle=\frac{1}{3}\left\langle K_{\text {total }}\right\rangle \simeq \beta \cdot \alpha$. We have used $\alpha=1.62$ and $\beta=0.162$ leading to $\left\langle K_{\gamma}\right\rangle=0.26[8]$. The event was accepted if $E_{\gamma}>E_{t h}=6.3 \mathrm{TeV}$, otherwise we have repeated the procedure from the point of selection of $\sigma\left(E \Rightarrow E-3 E_{\gamma}\right)$ adding the new distance $x_{1}$ to the previous $x$ [9].

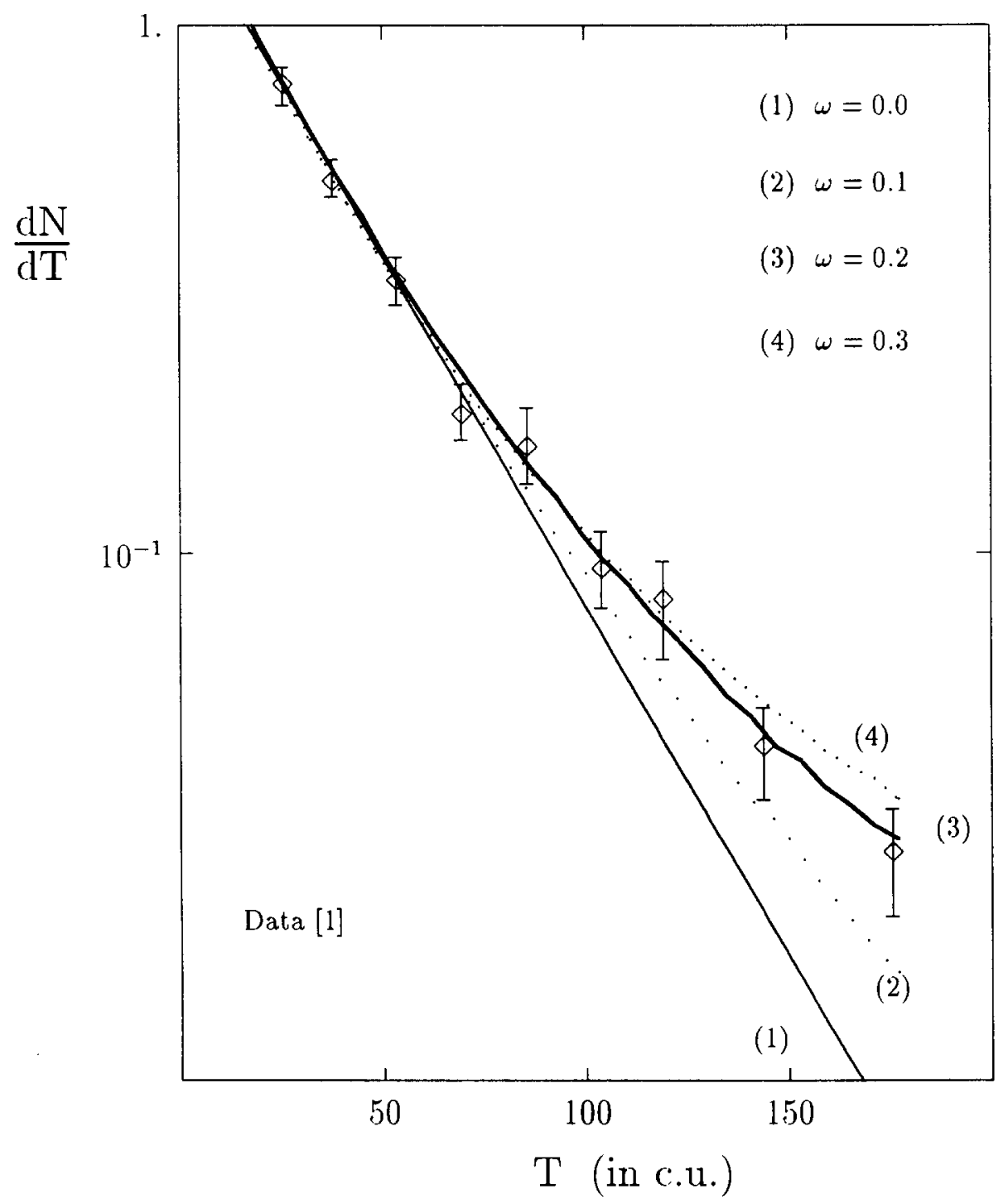

Figure 1: Depth distribution of cascades starting points in PAMIR lead chamber for different fluctuations of the interaction cross section as provided by the variance $\omega$ of the distribution of the cross section.

Results presented in Fig. 1 show that we can fit PAMIR data in a very reasonable way. The best agreement with data is obtained for the variance of the distribution of cros section $\omega=0.2$. As can be seen in Fig. 2 this value agrees also reasonably well with the highest energy values of $\omega$ presented in $[4,5]$ which were obtained from UA4 and TEVATRON data (cf. $[4,5]$ for details). Note that experimental points from accelerator data presented here come from $p p, p d$ and $p \bar{p}$ collisions whereas $\omega=0.2$ is extracted from PAMIR data under the tacit assumption that fluctuations in $p-P b$ are the same as 
in hadronic collisions (i.e., that internal configurations of projectile is frozen when it passes through the target nucleus). For this reason our $\omega_{P A M I R}=0.2$ should be regarded as lower limit of the "true" $\omega_{N N}$ at this energy.

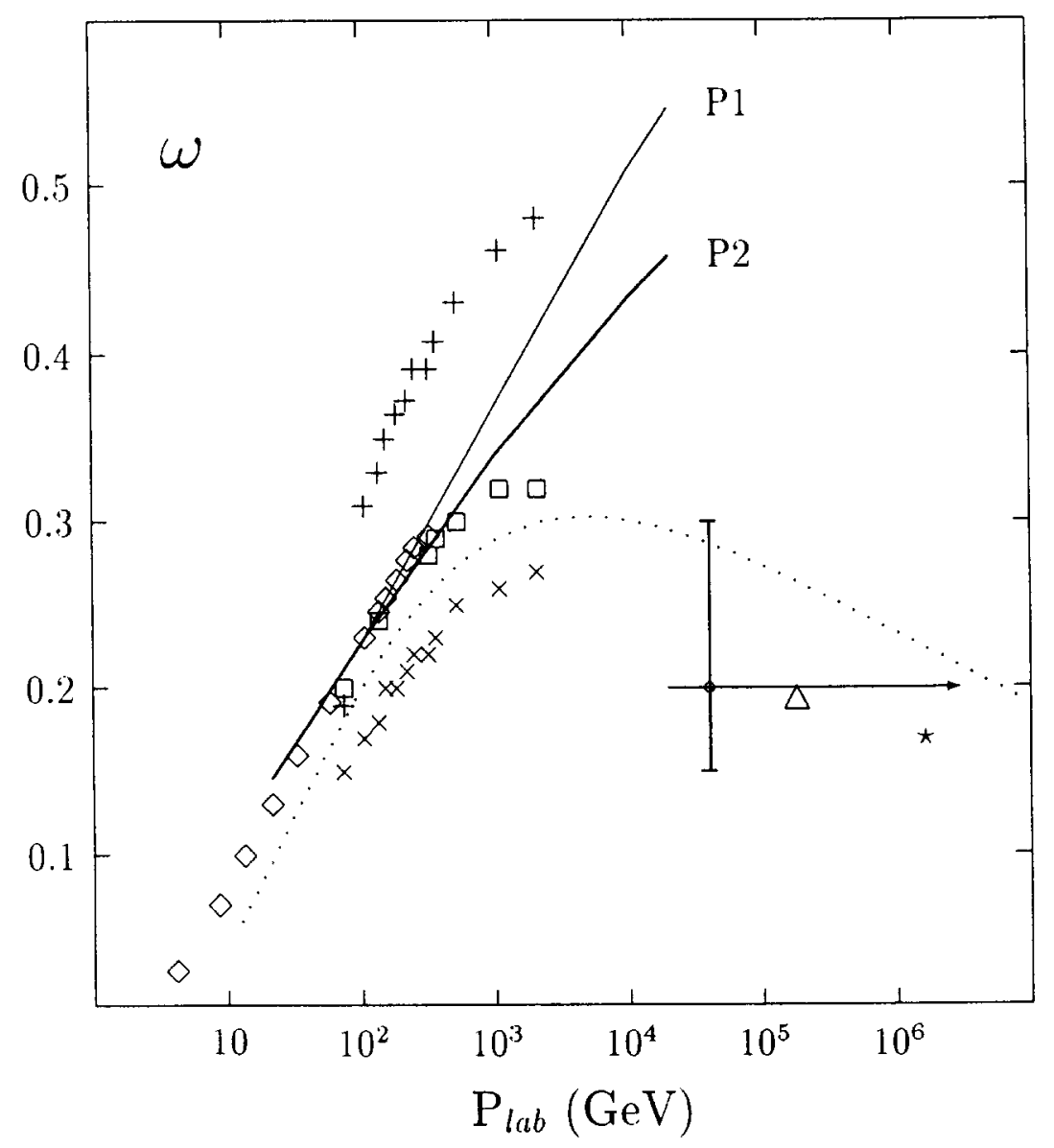

Figure 2: The energy dependence of the variance $\omega$ of the distribution of cross section obtained from different sources. The point with errorbars corresponds to our result with $\omega=0.2$ in Fig. 1 . All other points (except "star") and curves "P1" and "P2" are taken from Fig. 1 of [5], the "star" corresponds to TEVATRON data (ref. 33 in [5]). The dotted curve is our estimation of the possible behaviour of $\omega$ at high energies, cf. text for details.

Note that fluctuations considered here would also result in a small enhancement of $d N / d T$ at low $T$ However, it is most probably unobservable because of the dominantion of contributions from cascades originated by $\gamma$ quanta which are impossible to eliminate (in fact, that is the reason that results of PAMIR [1] do not extend below $T \leq 30$ ).

On the basis of our results (Figs. 1 and 2) we can argue that fluctuations of cross sections should be regarded as yet another possible candidate for the explanation of "exotic" cosmic ray data, example of which was discussed above [10]. Unfortunately our cosmic ray data point has considerable uncertainty in energy (which is the usual weak point of this kind of experiments) and for a time being it bears big error bars (which can be reduced with the accumulation of new data $[1,2]$ ).

It is interesting to note that, combined with two other accelerator results shown in Fig. 2, our result suggests that fluctuations will not grow indefinitely with energy. In Fig. 2 we have presented after [5] two possible theoretical predictions for $\omega(s)$ : "P1" $-\omega(s) \sim \ln s$, i.e., growing with energy, which is consistent with the single critical Pomeron exchange and "P2" $-\omega(s)=b-c / s^{\varepsilon}$, i.e., asymptotically constant, which 
is suggested by supercritical Pomeron exchange (cf. [5] for details). However, the last three points in Fig. 2 strongly suggest that $\omega(s)$ behaves likely as indicated by the dotted curve. This curve is obtained under simple assumption (which physical context is at present unknown) that cross section $\sigma$ contains two parts: (i) - an energy independent but strongly fluctuating $\sigma_{0}$ (taken as $39 \mathrm{mb}$ with $\omega_{0}=0.06 \ln (s / 4)$ as in [5] which fits the low energy data) and (ii) - growing with energy and non-fluctuating $\sigma_{1}(s)=0.29 \ln ^{2}(s / 70)$ with $\omega_{1}=0$. The resultant $\omega(s)$ is now given by

$$
\omega(s)=\omega_{0}(s) \cdot \frac{\left\langle\sigma_{0}\right\rangle^{2}}{\left\langle\sigma_{0}+\sigma_{1}(s)\right\rangle^{2}}
$$

and with $\sigma_{1}(s)$ chosen to fit the high energy data on cross section it gives the dotted curve in Fig. 2. It grows first according to $\omega_{0}(s)$ but at large energies $\sqrt{s}$ the energy grow of denominator in eq. (5) prevails and asymptotically $\omega(s) \sim$ const $/ \ln ^{3} s$ there $[11]$

As was already mentioned, fluctuating cross sections can be regarded as "non-exotic" variation of the second hypothesis but there is still room for some charm admixture. More new data and some new analyses of the old data from the point of view of the possibility of fluctuations in cross sections are therefore needed [12]. This would be especially welcome because fluctuations of $\sigma$ equivalent to the appearance of long flying component with $\lambda$ of the order of a few hundreds of $\mathrm{g} / \mathrm{cm}^{2}$ can result in strong fluctuations in the transverse and longitudinal development of the atmospheric families and in the phenomenon of alignement of structural objects of families [13].

We would like to thank Prof. M.Szczekowski for very useful and illuminating discussion

\section{References}

[1] S.A.Slavatinsky, in Proc. of $V I I^{\text {th }}$ Very Hifg Energy Cosmic-Ray Interactions, Ann Arbor, MI, June 21-27, 1992, AIP Conference Proceedings 276, ed. L. Jones, AIP, New York 1993, p. 3.

[2] Cf. V.I.Yakovlev, ibidem, p. 154.

[3] In this experiment the lead chamber with thickness $110 \mathrm{~cm}$ with area $30 \mathrm{~m}^{2}$ was exposed for 2 years and the total number of nuclear electromagnetic cascades (NEC) registered was equal 900 for the threshold (the smallest energy of the electromagnetic component recorded) $E_{t h}=6.3 \mathrm{TeV}$ (in LAB) (cf. [1] for more details).

[4] H.Heiselberg et al., Phys. Rev. Lett. 67, 2946 (1991).

[5] B.Blättel et al., Phys. Rev. D47, 2761 (1993).

[6] L.T.Baradzei et al. (Chacaltaya and Pamir Collab.), Nucl. Phys. B370, 365 (1992).

[7] In calculations we used the value of hadron flux at Pamir mountain altitude ( $4.3 \mathrm{~km}$ above sea level) equal to $578 \mathrm{~g} / \mathrm{cm}^{2}$ (what leads to $A=80 \mathrm{~m}^{-2} \mathrm{yr}^{-1} \mathrm{sr}^{-1} \mathrm{TeV}^{-1}$ and corresponds to $N(>20 \mathrm{TeV})=$ $\left.40 \mathrm{~m}^{-2} \mathrm{yr}^{-1} \mathrm{sr}^{-1}=1.3 \cdot 10^{-10} \mathrm{~cm}^{-2} \mathrm{~s}^{-1} \mathrm{sr}^{-1}[6]\right)$.

[8] B.L.Kanevsky et al., $19^{\text {th }}$ Int. Cosmic Ray Conf., 6, La Jolla (1985) 31.

[9] Notice that we have neglected here the apparent energy dependence of the inelasticity $K=K(E)$ (cf. for example: Yu.M.Shabelski, R.M.Weiner, G.Wilk and Z.Wlodarczyk, J.Phys. G18, 1281 (1992)) It can be shown that it does not change the results for the $\omega$

[10] There are also other results which presumably can also be explained in this way, for example: nonexponential character of absorption of the EAS (Extensive Air Showers) in atmosphere or the so called "Tien-Shan effect" which consist on the existence of the long flying component in the ionizing calorimeter with lead absorbers, cf. [2] for more details.

[11] Such behaviour could arise, for example, if $\sigma_{1}(s)$ is caused by the creation or activation of some new degrees of freedom the number $N$ of which increases with energy (gluons?). In this case asymtotically one can expect that $\omega$ decreases as $\sim 1 / N^{2}$. We plan to address this problem elsewhere 
[12] In fact there is new PAMIR experiment going on devoted to measurement of the charm production cross section in e new X-ray emulsion chamber with large air gap $(2.5 \mathrm{~m})$ between two blocks of lead. cf. L.G.Sveshnikova and O.P.Strogova, $23^{\text {th }}$ Int. Cosmic Ray Conf. 4, Calgary (1993) 33 (contributed paper).

[13] Z. Wlodarczyk, rapporteur talk given at the $23^{\text {th }}$ Int. Cosmic Ray Conf., Calgary, July 10-30, 1993, to be published in proc., eds. R.B.Hicks et al., World Scientific 1994. 